TASK COMPLETION REPORT FOR UPDATE FXTPTM

Robert G. Steinke, TSA-10

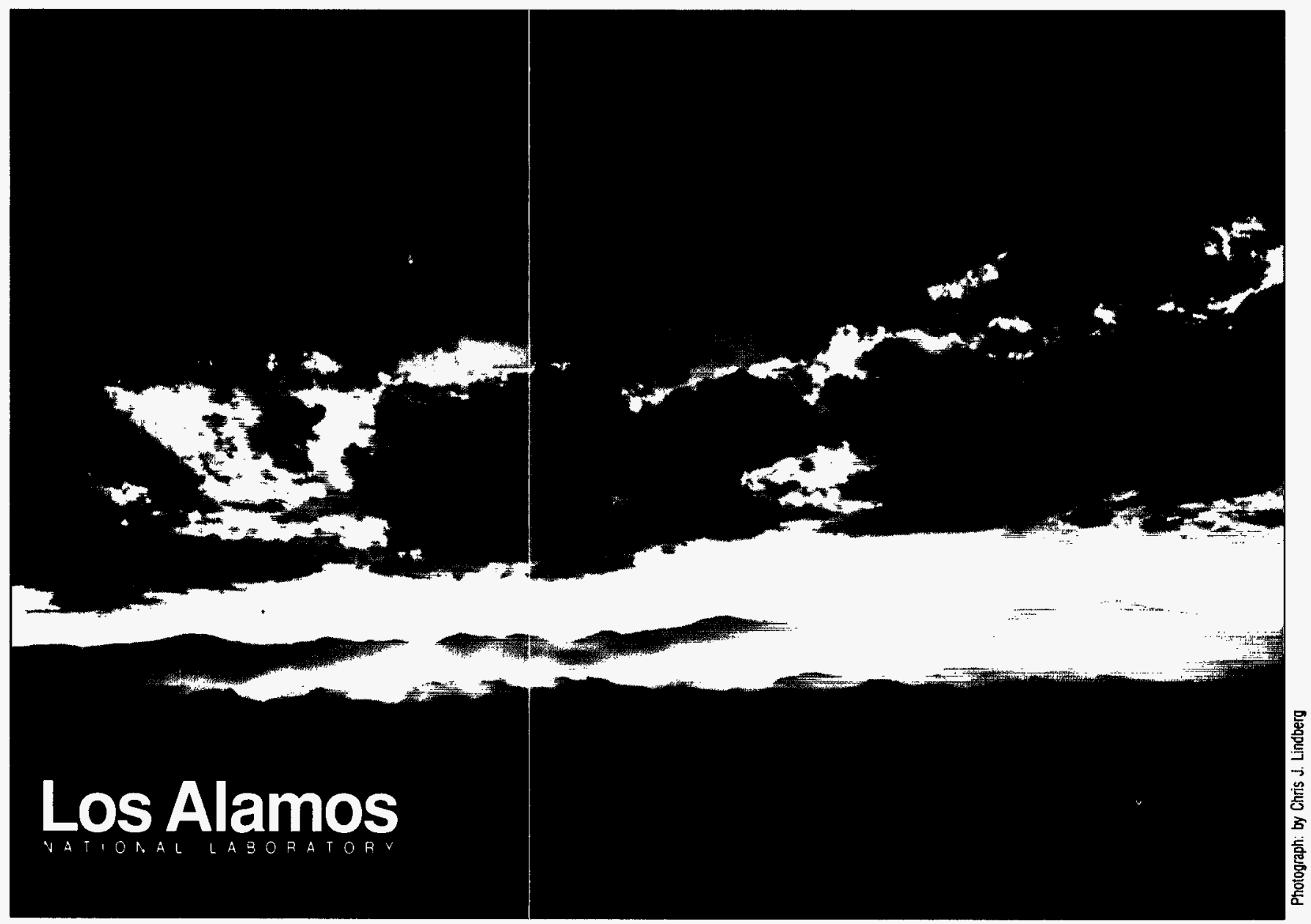

Los Alamos National Laboratory, an affirmative action/equal opportunily employer, is operated by the University of Californin for the U.S. Department of Energy under contract published form of this contribution, or to allow others to do so, for U.S. Government purposes. The Lont retains a nonexclusive, royalty-free as work performed under the auspices of the U.S. Department of Energy, Los Alamos National Laboratory strongly supports academic freedom and a researcher's right to publish therefore, the Laboratory as an institution does not endorse the viewpoint of a publication or guarantec its technical correctness. 


$$
\text { LA. UR }--98-104
$$

\section{TASK COMPLETION REPORT FOR UPDATE FXTPTM}

$$
\text { by }
$$

Robert G. Steinke

August 29, 1997

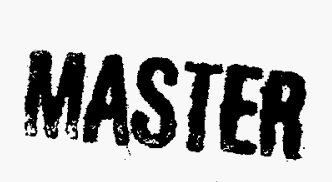

Gunidion of Mis Documen to entumed 


\section{DISCLAMMER}

This report was prepared as an account of work sponsored by an agency of the United States Government. Neither the United States Government nor any agency thereof, nor any of their employees, makes any warranty, express or implied, or assumes any legal liability or responsibility for the accuracy, completeness, or usefulness of any information, apparatus, product, or process disclosed, or represents that its use would not infringe privately owned rights. Reference herein to any specific commercial product, process, or service by trade name, trademark, manufacturer, or otherwise does not necessarily constitute or imply its endorsement, recommendation, or favoring by the United States Government or any agency thereof. The views and opinions of authors expressed herein do not necessarily state or reflect those of the United States Government or any agency thereof. 


\section{DISCLAIMER}

Portions of this document may be illegible electronic image products. Images are produced from the best available original document. 


\title{
TASK COMPLETION REPORT \\ FOR UPDATE FXTPTM
}

\author{
Robert G. Steinke
}

\begin{abstract}
Update FXTPTM corrects a trip pointer error in subroutine TIMCHK that prevented the trip-initiated, problem-termination feature from functioning in TRAC-P.
\end{abstract}

\subsection{INTRODUCTION}

Knolls Atomic Power Laboratory reported that the trip-initiated, problem-termination feature was not functioning in TRAC-P, Version 5.4.15. A listing of the modified input data for the DRAIN standard test problem and the TRCMSG file output was provided that demonstrated that the TRAC-P calculation did not terminate when the problem-termination designated trip's set status was set to $\mathrm{ON}_{\text {forward. }}$. The assigned task was to investigate and correct this nonfunctioning feature in TRAC-P, Version 5.4.28.

\subsection{UPDATE FXTPTM}

The cause of the trip-initiated, problem-termination feature not functioning was traced to a misdefined pointer variable in subroutine TIMCHK. When update UNITS programmed TRAC-PF1/MOD2, Version 5.4, to provide SI/English units input/output, all A-array pointer variables for the trip data needed to be shifted to provide additional storage space in the control-parameter data section for controlblock and trip-signal, user-defined, units-name labels. The trip pointer variable in subroutine TIMCHK did not have this shift modification done to it by update UNITS, and none of the UNITS test problems exercised this trip-initiated, problemtermination feature. Appendix A shows a listing of update FXTPTM (FiX TriP TerMination) that corrects the definition of this pointer variable in subroutine TIMCHK.

\subsection{TESTING}

The DRAIN, MARVIK, and ZIONPWR standard test problems were modified to test the trip-initiated, problem-termination feature correction of update FXTPTM. Appendix B shows a listing of portions of their TRACIN files that were modified, and the end portion of their terminal-output listings where the controlling trip, after its set status was set to $\mathrm{ON}_{\text {forward }}$, terminated the calculation at the start of the 
next timestep. The DRAIN test problem was modified in the same manner as the KAPL test problem. The MARVIK test problem defined two trips where the first trip, when set to $O N_{\text {forward }}$ at $10 \mathrm{~s}$, only generated a restart data dump; the second trip, when set to $O N_{\text {forward }}$ at $15 \mathrm{~s}$, generated a restart data dump and terminated the calculation. The ZIONPWR test problem used an existing pump-controlling trip to terminate the calculation when the pump trip's set status was set to $\mathrm{ON}_{\text {forward }}$. All three of these test problems show that the trip-initiated, restart-dump and problemtermination feature in TRAC-P is now functioning correctly.

\subsection{CONCLUSIONS}

The trip-initiated, problem-termination feature in TRAC-P, Version 5.4.28 has been fixed by update FXTPTM. The feature was not functioning because update UNITS in TRAC-PF1/MOD2, Version 5.4 did not shift a trip pointer variable in subroutine TIMCHK.

\section{ACKNOWLEDGMENT}

The author wishes to thank Richard J. Smith for valuable discussions during the development and testing of this TRAC-P update. 
*ident fxtptm

\section{APPENDIX A \\ Listing of Update FXTPTM}

*/

$* 1$

* File \& Update Ident name (must be the same): fxtpum

*/ TSHOOTER identifier:

$* /$

* / name of

* $/$ SUBROUTINE

* / OR COMDECK

* CHANGED

$* /$

*/ TIMCHK A pointer variable definition is corrected.

$* 1$

* / subroutine timchk changes

*delete ifrd. 628

Date developed: $6 / 2 / 97$

Acknowledgements: KAPL reported this error

Update to be applied to MOD2 _ $X_{\text {_ }}$ or other :

CFS file path: $/ 073417$

Purpose of update: Update fxtptm (Fix TriP TerMination) corrects a pointer error in subroutine TIMCHK that prevented a trip when set on from terminating a TRAC-P calculation.

Basis for update:

A designated trip for terminating a TRAC-P calculation did not terminate the calculation when its set status changed from OFF to ON.

Dependencies on other updates: none

Justification of non-ANSI coding: N.A.

Is this a NULI update? YES__ NO _ X_

Does this update generate new unit labels? YES__ NO_X_

Does the TRAC I/O change because of this update? YES - NO _ yes, then submit the necessary manual changes.

Does this change require that any of the TRAC support codes, e.g. TRACT, GOCNVRT, or EXCON, be changed? YES _ NO _X

, then include a description of the necessary changes. support codes.

Does the TRAC User's Guide, Theory Manual, or Programmer's Guide change because of this update? YES NO _ $\mathrm{X}_{-}$

* alete ifra. 628

DESCRIPTION OF CHANGE 


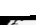

$$
\begin{aligned}
& 1=1 \operatorname{cnt} l+10+6 * i a(1 \operatorname{cn} t 1+5)+7 * i a(1 \operatorname{cnt} 1+1)+17 * i a(1 \operatorname{cn} t l+2)+i a(1 \operatorname{cn} t 1+3) \\
& 1+6 * i a(l \ln t l+2)+i a(1 \operatorname{cnt} l+4)+5 * i a(l \ln t l+6)-80
\end{aligned}
$$

* compile timchk

* 1 


\section{APPENDIX B \\ Portions of the TRACIN File and Terminal Listings From the DRAIN, MARVIK, and ZIONPWR Test Problems}

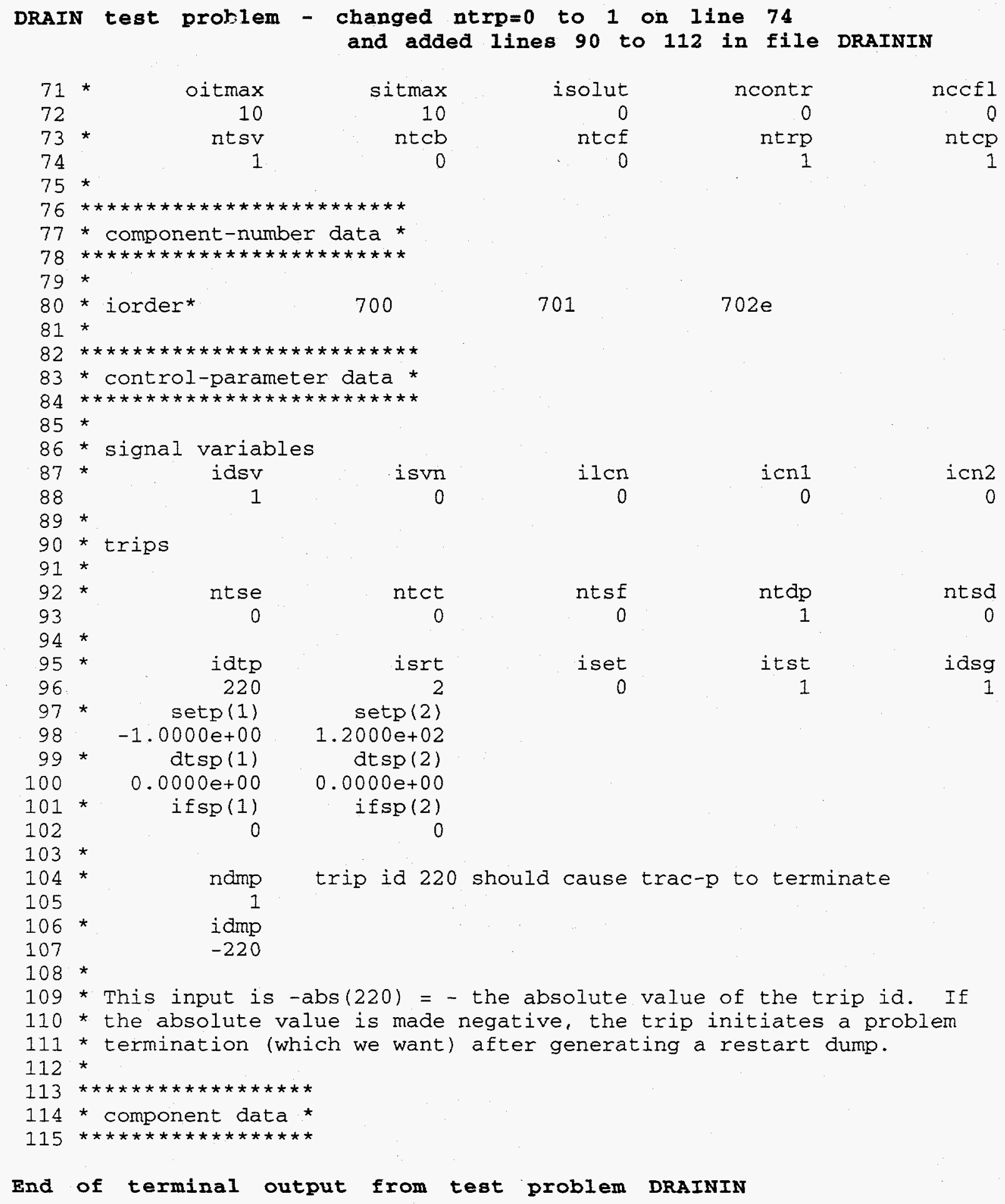




\begin{tabular}{|c|c|c|c|c|c|c|c|}
\hline 1910 & 91.568514 & 0.100000 & 2 & 0.2111 & 0.0000 & 1 & 700 \\
\hline 1920 & 92.568514 & 0.100000 & 2 & 0.2764 & 0.0000 & 1 & 700 \\
\hline 1930 & 93.568514 & 0.100000 & 2 & 0.2843 & 0.0000 & 1 & 700 \\
\hline 1940 & 94.568514 & 0.100000 & 2 & 0.2765 & 0.0000 & 1 & 700 \\
\hline 1947 & 95.268514 & 0.100000 & 2 & & & & \\
\hline 1950 & 95.568514 & 0.100000 & 3 & 0.0334 & 0.0000 & 1 & 700 \\
\hline 1960 & 96.568514 & 0.100000 & 3 & 0.0334 & 0.0000 & 1 & 700 \\
\hline 1970 & 97.568514 & 0.100000 & 3 & 0.0334 & 0.0000 & 1 & 700 \\
\hline 1980 & 98.568514 & 0.100000 & 3 & 0.0334 & 0.0000 & 1 & 700 \\
\hline 1990 & 99.568514 & 0.100000 & 3 & 0.0334 & 0.0000 & 1 & 700 \\
\hline 1997 & 100.268514 & 0.100000 & 2 & & & & \\
\hline 2000 & 100.568514 & 0.100000 & 2 & 0.0334 & 0.0000 & 1 & 700 \\
\hline 2010 & 101.568514 & 0.100000 & 3 & 0.0334 & 0.0000 & 1 & 700 \\
\hline 2020 & 102.568514 & 0.100000 & 2 & 0.0334 & 0.0000 & 1 & 700 \\
\hline 2030 & 103.568514 & 0.100000 & 3 & 0.0334 & 0.0000 & 1 & 700 \\
\hline 2040 & 104.568514 & 0.100000 & 2 & 0.0334 & 0.0000 & 1 & 700 \\
\hline 2047 & 105.268514 & 0.100000 & 3 & & & & \\
\hline 2050 & 105.568514 & 0.100000 & 3 & 0.0225 & 0.0000 & 1 & 700 \\
\hline 2060 & 106.568514 & 0.100000 & 2 & 0.0017 & 0.0000 & 1 & 700 \\
\hline 2070 & 107.568514 & 0.100000 & 3 & 0.0225 & 0.0000 & 1 & 700 \\
\hline 2080 & 108.568514 & 0.100000 & 2 & 0.0017 & 0.0000 & 1 & 700 \\
\hline 2090 & 109.568514 & 0.100000 & 3 & 0.0226 & 0.0000 & 1 & 700 \\
\hline 2097 & 110.268514 & 0.100000 & 2 & & & & \\
\hline 2100 & 110.568514 & 0.100000 & 2 & 0.0051 & 0.0000 & 1 & 700 \\
\hline 2110 & 111.568514 & 0.100000 & 2 & 0.0011 & 0.0000 & 1 & 700 \\
\hline 2120 & 112.568514 & 0.100000 & 2 & 0.0030 & 0.0000 & 1 & 700 \\
\hline 2130 & 113.568514 & 0.100000 & 2 & 0.0042 & 0.0000 & 1 & 700 \\
\hline 2140 & 114.568514 & 0.100000 & 2 & 0.0060 & 0.0000 & 1 & 700 \\
\hline 2147 & 115.268514 & 0.100000 & 2 & & & & \\
\hline 2150 & 115.568514 & 0.100000 & 2 & 0.0081 & 0.0000 & 1 & 700 \\
\hline 2160 & 116.568514 & 0.100000 & 2 & 0.0110 & 0.0000 & 1 & 700 \\
\hline 2170 & 117.568514 & 0.100000 & 2 & 0.0151 & 0.0000 & 1 & 700 \\
\hline 2180 & 118.568514 & 0.100000 & 2 & 0.0215 & 0.0000 & 1 & 700 \\
\hline 2190 & 119.568514 & 0.100000 & 2 & 0.0328 & 0.0000 & 1 & 700 \\
\hline
\end{tabular}
at $120.000000 \mathrm{~s}$, the trip 220 signal crossed setpoint $\mathrm{s} 2=1.200000 \mathrm{E}+02$ * at $120.068514 \mathrm{~s}$, the trip 220 signal is $1.200685 \mathrm{E}+02$ *
at $120.068514 \mathrm{~s}$, trip 220 is reset from 0 to 1 with a set status of on-forward restart dump generated at problem time $120.168514 \mathrm{~s}$ after 2196 time steps time-step problem time-step outer-it. cpu courant numbers sets location number time (s) size (s) number time (s) 1-d 3 -d flag cmp cell $\begin{array}{llll}2196 & 120.168514 & 0.100000 & 2\end{array}$

end of problem

computative timing statistics

cpu time is $s$

Note: IEEE floating-point exception traps enabled: overflow; division by zero; invalid operation;

see the Numerical Computation Guide, ieee_handler(3M)

castor:/export/castor1/rgs/trac> mv trcout drainout 
MARVIR test problem - changed ntsv=0 to 1 and ntrp=0 to 2 on 1 ine 91 and added lines 99 to 137 in file MARVIRIN

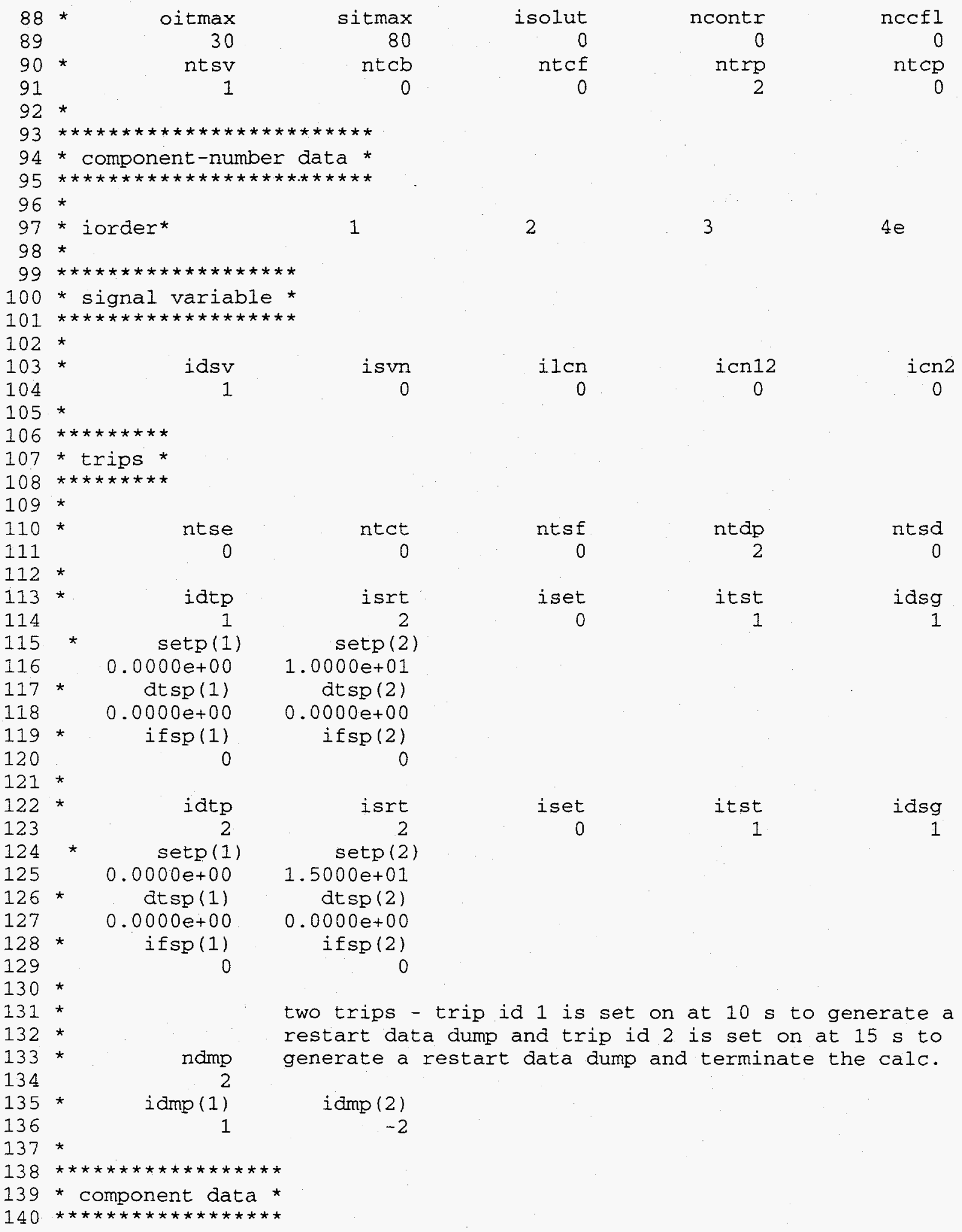


input data is being processed

h20 properties are used

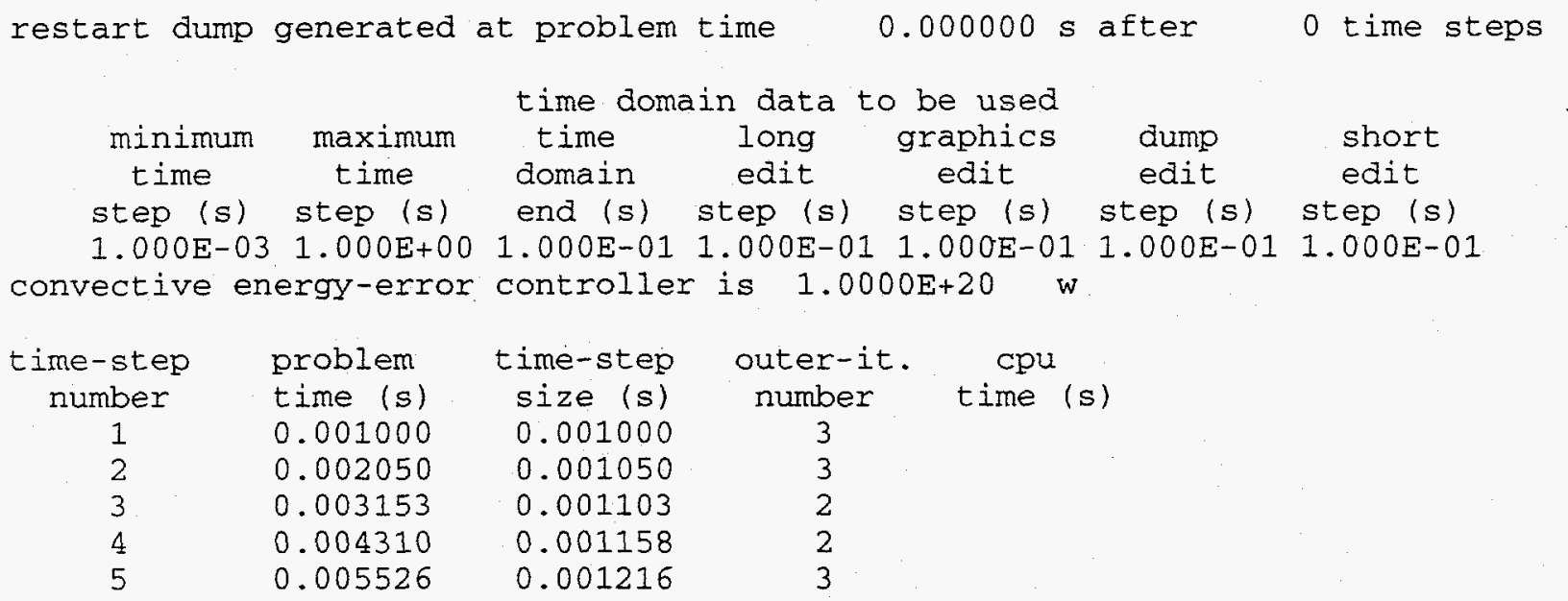

restart dump generated at problem time $\quad 0.101200 \mathrm{~s}$ after 38 time steps

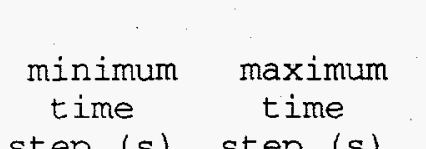

step (s) step (s)

$1.000 \mathrm{E}-03 \quad 1.000 \mathrm{E}+00$

convective energy-error

$\begin{array}{cc}\begin{array}{c}\text { time-step } \\ \text { number }\end{array} & \begin{array}{c}\text { problem } \\ \text { time (s) }\end{array} \\ 88 & 1.122178 \\ 102 & 2.217262 \\ 110 & 3.273677 \\ 116 & 4.385453 \\ 120 & 5.329539 \\ 124 & 6.477082 \\ 128 & 7.790086 \\ 131 & 8.935421 \\ 134 & 10.261289\end{array}$

time domain data to be used

$\begin{array}{cccc}\text { time long graphics dump } & \text { short } \\ \text { domain edit edit } & \text { edit } & \text { edit }\end{array}$

end (s) step (s) step (s) step (s) step (s) $5.600 \mathrm{E}+015.000 \mathrm{E}+00 \quad 5.000 \mathrm{E}-01 \quad 1.000 \mathrm{E}+01 \quad 1.000 \mathrm{E}+00$ controller is $1.0000 \mathrm{E}+20 \mathrm{~W}$

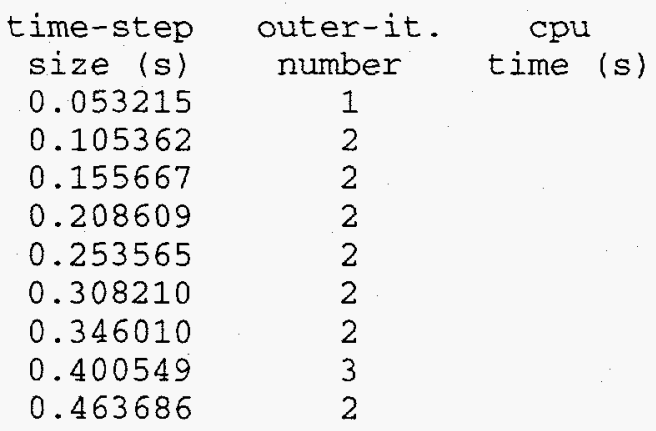

restart dump generated at problem time $10.261289 \mathrm{~s}$ after 134 time steps

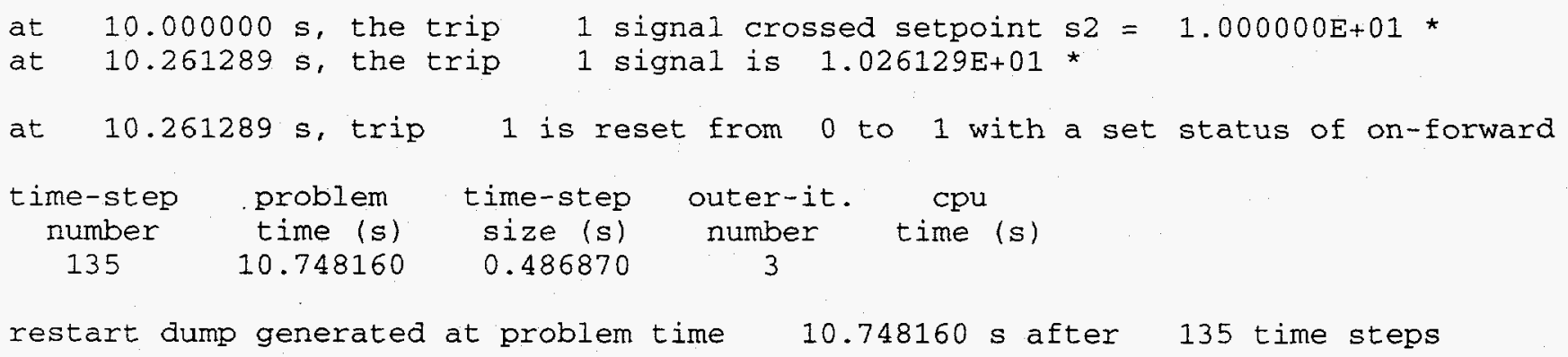




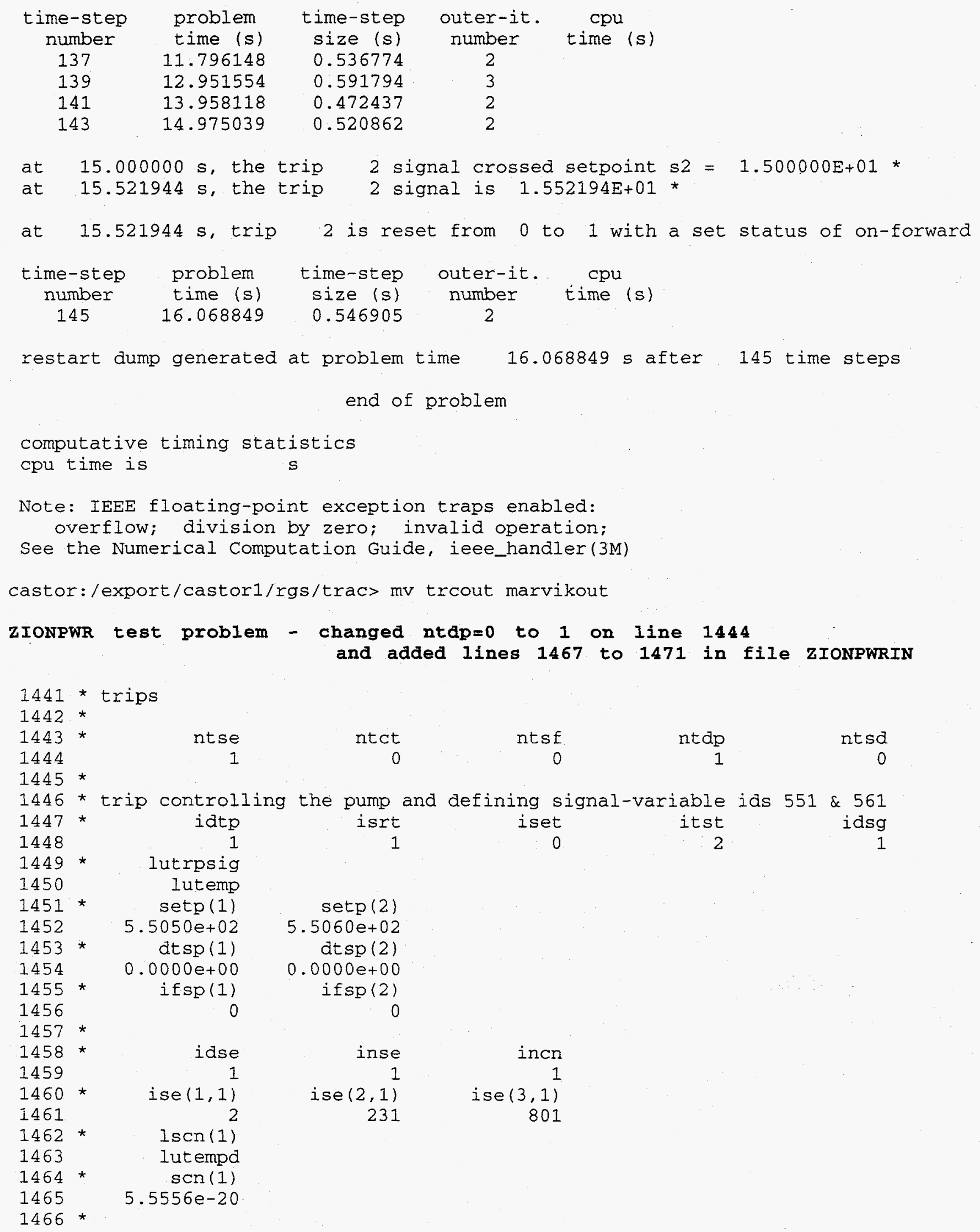




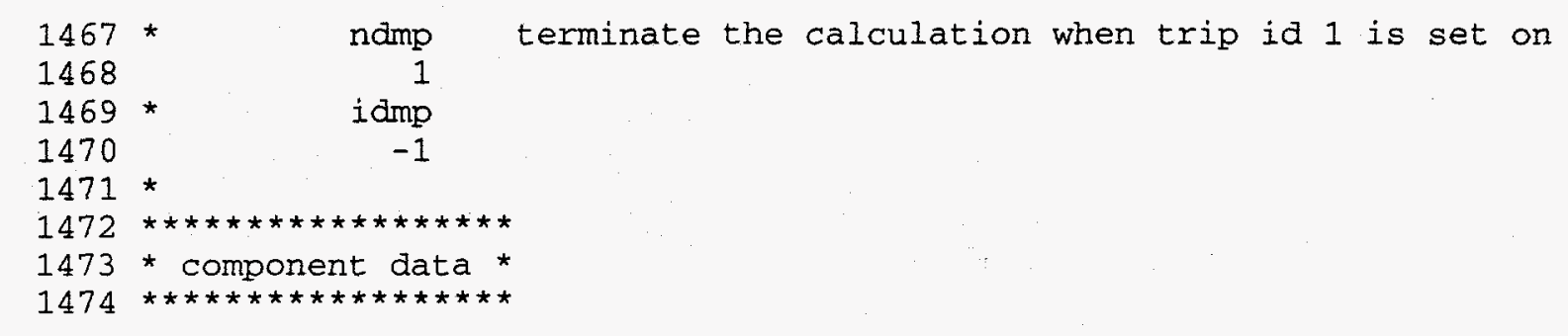

\section{End of terminal output from test problem zIONPWRIN}
time-step problem time-step outer-it. cpu courant numbers sets location number time (s) size (s) number time (s) 1-d 3-d flag cmp cell
5
0.005000
0.001000
2

\begin{tabular}{ccccc}
\multicolumn{5}{c}{ time domain data to be used } \\
minimum maximum & time & long & graphics dump & short \\
time & time & domain & edit & edit
\end{tabular}

step (s) step (s) end (s) step (s) step (s) step (s) step (s)

1.000E-03 2.500E-01 2.000E+01 2.100E+01 5.000E-01 2.100E+01 2.100E+01

convective energy-error controller is $1.0000 \mathrm{E}+20$ w

\begin{tabular}{|c|c|c|c|c|c|c|c|c|c|}
\hline time-step & problem & time-step & outer-it & cpu & courant & numbers & sets & $10 \mathrm{C}$ & atio \\
\hline number & time (s) & size (s) & number & time (s) & $1-d$ & $3-d$ & flag & $\mathrm{cmp}$ & \\
\hline 10 & 0.010000 & 0.001000 & 2 & & 0.0053 & 0.0053 & 0 & 2 & \\
\hline 20 & 0.022027 & 0.001551 & 2 & & 0.0080 & 0.0080 & 0 & 2 & \\
\hline 30 & 0.041539 & 0.002527 & 2 & & 0.0137 & 0.0138 & 0 & 3 & \\
\hline 40 & 0.073323 & 0.004116 & 2 & & 0.0219 & 0.0219 & 0 & 2 & \\
\hline 50 & 0.125095 & 0.006705 & 1 & & 0.0357 & 0.0357 & 0 & 2 & \\
\hline 60 & 0.209427 & 0.010921 & 1 & & 0.0582 & 0.0582 & 0 & 2 & \\
\hline 70 & 0.346794 & 0.017790 & 1 & & 0.0948 & 0.0950 & 0 & 3 & \\
\hline 80 & 0.570551 & 0.028978 & 1 & & 0.1545 & 0.1554 & 0 & 3 & \\
\hline 90 & 0.935027 & 0.047201 & 1 & & 0.2516 & 0.2544 & 0 & 3 & \\
\hline 100 & 1.528721 & 0.076886 & 1 & & 0.4099 & 0.4169 & 0 & 3 & \\
\hline 110 & 2.495786 & 0.125239 & 1 & & 0.6678 & 0.6854 & 0 & 3 & \\
\hline
\end{tabular}

\footnotetext{
at $\quad 3.894929 \mathrm{~s}$, the trip 1 signal crossed setpoint $\mathrm{s} 1=5.505000 \mathrm{E}+02 \mathrm{k}$ at $4.071032 \mathrm{~s}$, the trip 1 signal is $5.504990 \mathrm{E}+02 \mathrm{k}$

at $4.071032 \mathrm{~s}$, trip 1 is reset from 0 to 1 with a set status of on-forward

time-step problem time-step outer-it. cpu courant numbers sets location

$\begin{array}{ccccccccc}\text { number } & \text { time (s) } & \text { size (s) } & \text { number } & \text { time (s) } & 1-d & 3-d & \text { flag cmp cell } \\ 120 & 4.071032 & 0.204002 & 1 & & 1.0879 & 1.1331 & 1 & 3\end{array}$ 120 4.071032

$0.204002 \quad 1$

1.0879

restart dump generated at problem time $4.275034 \mathrm{~s}$ after 121 time steps

time-step problem time-step outer-it. cpu courant numbers sets location number time (s) size (s) number time (s) $1-d \quad 3-d$ flag cmp cell 121 4.275034

0.204002 1

end of problem

computative timing statistics

cpu time is $\mathrm{s}$

Note: IEEE floating-point exception traps enabled: overflow; division by zero; invalid operation;
} 


\begin{abstract}
See the Numerical Computation Guide, ieee_handler(3M)
castor:/export/castor1/rgs/trac> mv trcout zionpwrout
\end{abstract}

